\title{
ON THE TOPOLOGICAL ENTROPY OF GREEN INTERVAL MAPS
}

\section{J. BOBOK}

Received February 24, 2000 and, in revised form, November 27, 2000

\begin{abstract}
We investigate the topological entropy of a green interval map. Defining the complexity we estimate from above the topological entropy of a green interval map with a given complexity. The main result of the paper — stated in Theorem 0.2 — should be regarded as a completion of results of [4].
\end{abstract}

\section{Introduction and main result}

The purpose of this paper is to evaluate the topological entropy of green interval maps. The topological entropy provides a numerical measure for the complexity of such one-dimensional dynamical systems and our aim is to describe this complexity by means of combinatorics. A particular case of a system given by a $P$-monotone map $f_{P}$ for a green cycle $P$ was investigated in [4]. Since a green interval map is not a uniform limit of such special green (Markov) maps, our Theorem 0.2 completes the result of [4].

1991 Mathematics Subject Classification. 26A18, 37B40.

Key words and phrases. Interval map, topological entropy, symbolic dynamics.

Research supported by GA of Czech Republic, the contract n. 201/00/0859 and by grant CEZ J04/98/210000010.

ISSN 1425-6908 (c) Heldermann Verlag. 


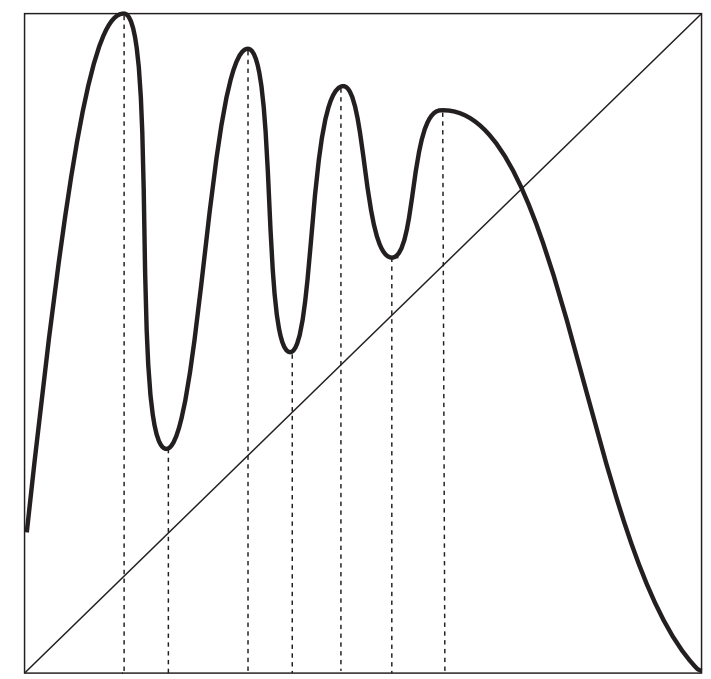

Figure 1: The green map $f ; l=3$

Green map ([2]). A continuous piecewise monotone map $f:[0,1] \rightarrow$ $[0,1]$ is said to be green if $f(0)>0, f(1)=0$ and it has an odd number $2 l+1$ of turning points $a_{1}<a_{2}<\cdots<a_{2 l+1}$ in $(0,1)$ and a unique fixed point $b>a_{2 l+1}$ such that (see Figure 1)

$$
f(0)<f\left(a_{2}\right)<\cdots<f\left(a_{2 l}\right)<b<f\left(a_{2 l+1}\right)<\cdots<f\left(a_{1}\right)=1 .
$$

Complexity. Let $f$ be a green map satisfying (1). If $f^{2}\left(a_{2 l+1}\right)<a_{1}$, the complexity $C(f)$ of $f$ is equal to $2 l+2$, otherwise $C(f)$ is defined as a minimal number $2 k \in\{2,4, \ldots, 2 l\}$ such that $f^{2}\left(a_{2 i+1}\right) \geq a_{2 i+1-2 k}$ for each $i=k, k+1, \ldots, l$.

Remark 0.1. The definition of complexity presented here slightly differs from the one given in [4].

By $\alpha(k)$ we denote the positive root of the polynomial equation

$$
(\alpha+1)^{k}\left(1+\sqrt{1+k^{2}}\right)^{k}+\alpha^{2}(\alpha-1)^{k} k^{k}\left(k-\sqrt{1+k^{2}}\right)=0 .
$$

The following theorem generalizes a result from [4].

Theorem 0.2. Let $f$ be a green map such that $C(f) \leq 2 k$. Then $\operatorname{ent}(f)<$ $\log \alpha(k)$. 
Remark 0.3. (i) As it was shown in [4], the bound $\log \alpha(k)$ is the least possible even for special green Markov maps with the complexity less than or equal to $2 k$, i.e. for any $\alpha<\alpha(k)$ there is a green Markov map of complexity less than or equal to $2 k$ with the entropy from $(\alpha, \alpha(k))$. It is stated there that each $\alpha(k)$ is irrational greater than 1 ,

$$
\lim _{k \rightarrow \infty} \frac{\alpha(k)}{k}=0 \quad \text { and } \quad \lim _{k \rightarrow \infty} \frac{\alpha(k)}{\sqrt{k}}=\infty .
$$

(ii) Using a method analogous to the one from [3] we can prove the inequality

$$
\operatorname{ent}(f) \geq \frac{1}{2} \log (C(f)-2) \text {. }
$$

\section{Lemmas and proofs}

We will use the symbolic dynamics [7]. As usually, for $m \in \mathbb{N}$ let us consider $N_{m}=\{1, \ldots, m\}$ as a finite space with the discrete topology, denote by $\Omega_{m}$ the infinite product space $\prod_{i=0}^{\infty} X_{i}$, where $X_{i}=N_{m}$ for all $i$. The shift map $\sigma: \Omega_{m} \rightarrow \Omega_{m}$ is defined by

$$
(\sigma(\omega))_{i}=\omega_{i+1}, i \in \mathbb{N} \cup\{0\} .
$$

A subset $\Omega$ of $\Omega_{m}$ is $\sigma$-invariant if $\sigma(\Omega) \subset \Omega$, the pair $(\Omega, \sigma)$ is transitive if there is a point $\omega \in \Omega$ such that $\overline{\left\{\sigma^{i}(\omega): i \in \mathbb{N}\right\}}=\Omega$. We write $\Omega(k)=$ $\left\{\left(\omega_{0}, \ldots, \omega_{k-1}\right): \omega \in \Omega\right\}$. The following proposition presents well-known results about topological entropy (for its definition see [6], [7]).

Lemma 1.1. [7] Let $\Omega, \Gamma \subset \Omega_{m}$ be closed and $\sigma$-invariant, $(\Gamma, \sigma)$ transitive, $\Omega \subset \Gamma$ and $\Omega \neq \Gamma$. Then $\operatorname{ent}(\sigma \mid \Omega)<\operatorname{ent}(\sigma \mid \Gamma)$.

For $I=[0,1]$ let $C(I)$ be a space of all continuous maps which map $I$ into itself. By $\mathcal{Q}$ we denote the set

$$
\left\{Q=\left\{q_{k}\right\}_{k=0}^{n}: 0=q_{0}<q_{1}<\cdots<q_{n-1}<q_{n}=1, n \in \mathbb{N}\right\} .
$$

A map $g \in C(I)$ is piecewise monotone if there is a $Q \in \mathcal{Q}$ such that the map $g$ is monotone (not necessarily strictly) on each interval $\left[q_{k-1}, q_{k}\right]$ given by $Q$. Such a $Q$ with the minimal cardinality is denoted by $T_{g}$. In the sequel the following notation will be useful. For $g \in C(I)$ and $Q=\left\{q_{k}\right\}_{k=0}^{n} \in \mathcal{Q}$, $Q(g)=\left\{x \in I: \forall i \in \mathbb{N} \cup\{0\} g^{i}(x) \notin Q\right\}$ and

$$
\Omega(Q(g))=\left\{\omega \in \Omega_{n}: \exists x \in Q(g) \forall i \in \mathbb{N} \cup\{0\} g^{i}(x) \in\left(q_{\omega_{i}-1}, q_{\omega_{i}}\right)\right\} .
$$

The following lemma shows the basic facts about relation of an entropy of a map $g \in C(I)$ and the symbolic dynamics given by $g$ and $Q \in \mathcal{Q}$. The references needed for the proofs are given. 


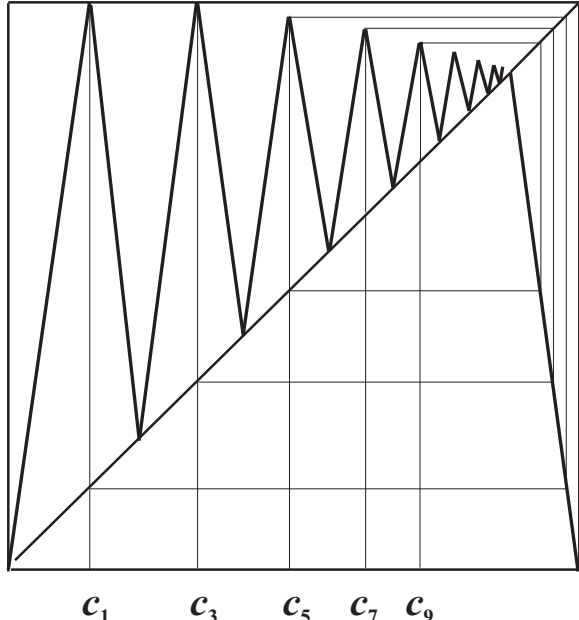

Figure 2: The map $F_{2}$

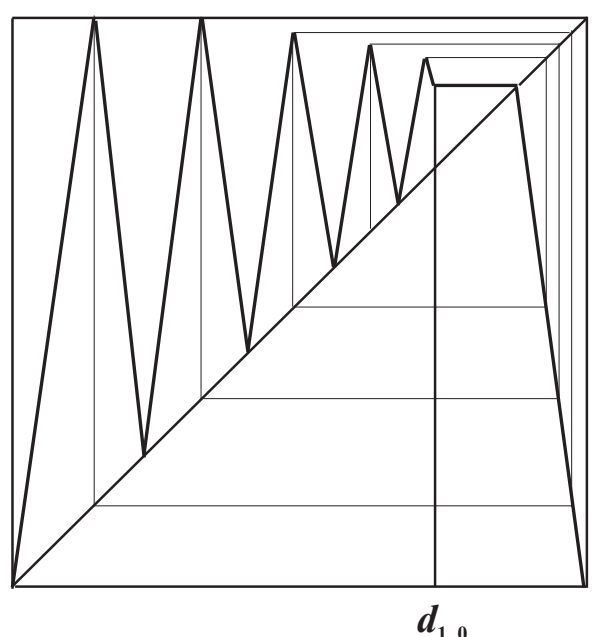

Figure 3: The map $G_{2,10}$

\section{Lemma 1.2.}

(i) [1], [5] For any $g \in C(I)$ and a g-invariant $Q \in \mathcal{Q}$,

$$
\operatorname{ent}(g) \geq \lim _{k} \frac{1}{k} \log \operatorname{card} \Omega(Q(g))(k) .
$$

(ii) [8], [1] Let $g \in C(I)$ be piecewise monotone. Then

$$
\operatorname{ent}(g)=\lim _{k} \frac{1}{k} \log \operatorname{card} \Omega\left(T_{g}(g)\right)(k) \text {. }
$$

In order to prove our Theorem we use a special map constructed in [4] (see Figure 2).

Lemma 1.3. Let $k \in \mathbb{N}$ be fixed. The value $\alpha=\alpha(k)$ given by (2) is the least one for which there exists a transitive $\alpha$-Lipschitz map $F_{k}:[0,1] \rightarrow$ $[0,1]$ with the following properties $(d=\alpha /(\alpha+1))$. For the sequence $\left\{c_{n}\right\}_{n=0}^{\infty}$ such that $0=c_{0}<c_{1}<c_{2}<c_{3}<\cdots<d, \lim c_{n}=d$,

(i) $F_{k}$ has a constant slope $\alpha$ on each interval $\left[c_{2 n-2}, c_{2 n-1}\right]$ and a slope $(-\alpha)$ on each $\left[c_{2 n-1}, c_{2 n}\right],[d, 1]$,

(ii) $F_{k}\left(c_{2 n-2}\right)=c_{2 n-2}$ for $n \geq 1, F_{k}^{2}\left(c_{2 n-1}\right)=c_{2(n-k)-1}$ for $n \geq k+1$,

(iii) $F_{k}\left(c_{2 n-1}\right)=1$ for $n \in\{1, \ldots, k\}$,

(iv) $\operatorname{ent}\left(F_{k}\right)=\log \alpha$.

Let us denote $F_{k}^{-1}(\{d\})=\left\{d_{1}<d_{2}<\cdots<d_{n}<\cdots\right\}$; for each $n \in \mathbb{N}$, $d_{n} \in\left(c_{n-1}, c_{n}\right)$. The continuous map $G_{k, m}:[0,1] \rightarrow[0,1]$ is defined by $G_{k, m}(x)=F_{k}(x), x \in\left[0, d_{m}\right] \cup[d, 1]$ and $G_{k, m}(x)=d, x \in\left[d_{m}, d\right]$ (see Figure 3). 
Let $f$ be the green map satisfying (1), for the sake of simplicity denote $G=G_{k, 2 l+2}$ and $F=F_{k}$. We have $T_{f}=\{0,1\} \cup\left\{a_{k}\right\}_{k=1}^{2 l+1}, T_{G}=\{0,1\} \cup$ $\left\{c_{k}\right\}_{k=1}^{2 l+1}$.

Put also $Q=\{0,1\} \cup\left\{c_{k}\right\}_{k=1}^{2 l+3}$. The following lemma describes the properties of the subsets (of $\Omega_{2 l+4}$ )

$$
\Omega(f)=\bar{\Omega}\left(T_{f}(f)\right), \Omega(G)=\bar{\Omega}\left(T_{G}(G)\right), \Omega(F)=\bar{\Omega}(Q(F)) .
$$

Note that all those subsets are closed and $\sigma$-invariant. By $\omega^{\prime}+\Omega$ we denote the set $\left\{\left(\omega_{1}^{\prime}+\omega_{1}, \omega_{2}^{\prime}+\omega_{2}, \ldots\right): \omega \in \Omega\right\}$.

\section{Lemma 1.4.}

(i) $\Omega(f) \subset \Omega(G)$.

(ii) $(1,1, \ldots)+\Omega(G) \subset \Omega(F),(\Omega(F), \sigma)$ is transitive and $(1,1, \ldots)+$ $\Omega(G) \neq \Omega(F)$.

Proof. By our assumption on the complexity of $f$ and the definition of $G$ it holds $G\left(\left(c_{k-1}, c_{k}\right)\right) \supset\left(c_{j-1}, c_{j}\right)$, whenever $f\left(\left(a_{k-1}, a_{k}\right)\right) \cap\left(a_{j-1}, a_{j}\right) \neq \emptyset$ and $k, j \in\{1, \ldots, 2 l+1\}$. This gives (i). Similarly it can be verified that $(1,1, \ldots)+\Omega(G) \subset \Omega(F)$; the transitivity of $(\Omega(F), \sigma)$ follows from that of $F$. The relation $(1,1, \ldots)+\Omega(G) \neq \Omega(F)$ is clear.

Now we are ready to give the proof of our main theorem.

Proof of Theorem 0.2. As above, $G=G_{k, 2 l+2}$ and $F=F_{k}$. Put $P=$ $Q \cup F(Q)$. Clearly, by Lemma 1.2(i) the entropy $\operatorname{ent}(F)$ is greater than or equal to $\lim _{k}(1 / k) \log \operatorname{card} \Omega(P(F))(k)$. Since $P \supset Q$ we have also

$$
\lim _{k} \frac{1}{k} \log \operatorname{card} \Omega(P(F))(k) \geq \lim _{k} \frac{1}{k} \log \operatorname{card} \Omega(Q(F))(k) .
$$

Now, it follows from Lemmas 1.4(ii), 1.1, 1.2(ii) and 1.4(i) that

$$
\lim _{k} \frac{1}{k} \log \operatorname{card} \Omega(Q(F))(k)>\operatorname{ent}(G) \geq \operatorname{ent}(f) .
$$

Summarizing, $\operatorname{ent}(F)=\log \alpha(k)>\operatorname{ent}(f)$. This proves the theorem. 


\section{References}

[1] Alsedá, L., Llibre, J., Misiurewicz, M., Combinatorial Dynamics and Entropy in Dimension One, Adv. Ser. Nonlinear Dynam. 5 (1993), World Sci., Singapore.

[2] Blokh, A. M., Rotation numbers for unimodal maps MSRI (1994), Preprint \#58-94.

[3] Blokh, A. M., Misiurewicz, M., Entropy of twist interval maps, Israel J. Math. 102 (1997), 61-99.

[4] Bobok, J., On entropy of patterns given by interval maps, Fund. Math. 162(1), 1999, $1-36$.

[5] Bobok, J., Kuchta, M., Invariant measures for maps of the interval that do not have points of some period, Ergodic Theory Dynam. Systems 14 (1994), 9-21.

[6] Bowen, R., Entropy for group endomorphisms and homogeneous spaces, Trans. Amer. Math. Soc. 153 (1971), 401-414.

[7] Denker, M., Grillenberger, Ch., Sigmund, K., Ergodic Theory on Compact Spaces, Lecture Notes in Math. 527 (1976), Springer-Verlag, Berlin.

[8] Misiurewicz, M., Szlenk, W., Entropy of piecewise monotone mappings, Studia Math. 67 (1980), 45-63.

JOzEF BOBOK

KM FSV ČVUT

THÁKUROVA 7

16629 Praha 6

Czech Republic

E-MAIL: ERASTUS@MBOX.CESNET.CZ 